\section{CLUSTER: A program for identifying recurrent clusters}

\author{
MURRAY ZANGEN, PHILIP ZIEGELBAUM, \\ and HERMAN BUSCHKE \\ Albert Einstein College of Medicine \\ Bronx, New' York 10461
}

A new way of analyzing organization in free recall (and other sequences) was recently proposed by Buschke (1976), who showed the clustering in free recall by identifying recurrent clusters of items that were recalled together and were demarcated by different items or clusters on different recall attempts. CLUSTER is a new program for analyzing sequential organization by finding recurrent groups and subgroups of items that occur together (regardless of order), delineating the specific clusters on each trial to show the development of organization and provide a basis for measuring organization in terms of such clustering.

Description. CLUSTER is an extension of Pellegrino's (1972) program for analyzing subjective organization from one trial to the next, extending Pellegrino's program search for recurrent clusters on any trial by comparing all sequences of items on every trial with those in all subsequent trials. Larger groups are broken up into component subgroups by comparing groupings in Trial 1 with those in Trials $2,3 \ldots \mathrm{N}$, groupings in Trial 2 with those in Trials $3,4 \ldots \mathrm{N}$, and so on until the smallest clusters have been identified. A cluster is identified as a group of items that are recalled together in any order on two or more trials and are demarcated by different items or clusters on different trials. Identifying clusters that recur on any trials shows the recurrence of clusters on more than just the next trial, so that the development and persistence of organization during learning can be analyzed in terms of the basic clustering and higher order organization of clusters.

While the main purpose of Pellegrino's (1972) program was to compute the amount of subjective organization in free recall learning (Pellegrino, 1971) without delineating specific clusters, our aim is to analyze the actual clustering on all trials, so that the nature of the

The authors gratefully acknowledge the assistance of the staff of the Albert Einstein College of Medicine Academic Computer Center. Dr. J. Pellegrino graciously supplied a prototype of this computer program. This work is supported by USPHS Grants MH-17733 to H. B. from NIMH, NS-03356 from NINDS, and HD-01799 from NICHHD. Requests for reprints should be sent to Herman Buschke, M.D., Department of Neurology, Albert Einstein College of Medicine, Bronx, New York 10461. clustering can be investigated, the development of organization over trials can be analyzed in terms of the clustering, and so that the amount of organization can be measured in terms of the recurrent clustering (Buschke, 1976). Consequently, the output of the present CLUSTER program provides, for each trial, a list of items as they were recalled-separated into higher order groups and component sub groups. The output also includes directories that show the clusters to which each item belongs, and the size, composition, frequency, and trials of occurrence of each cluster.

This recurrent clustering program also computes a new measure of organization for each trial (Buschke, 1976), by assigning to each cluster a score of $n-1$, where $n$ is the number of items in a cluster. This is the simplest measure of organization that will give the least higher score for a cluster of $n+1$ items than for a cluster of $n$ items, and the least higher score for a cluster of $2 n$ items than for two clusters of $n$ items. This measure of organization, which can be interpreted as the least number of connections necessary to link all of the items in a cluster, is linearly related to the number of items in a cluster, and provides a reasonable estimate of organization in terms of the recurrent clustering that seems to be the main characteristic of such organization.

Language. The program uses FORTRAN IV. Standard input/output procedures are utilized.

Computer. The computer used is an XDS Sigma 6 with $40 \mathrm{~K}$ words (160K bytes) of core memory.

Restrictions. Restrictions will vary with the amount of core storage actually available for the program and data at the user's installation. On the Sigma 6, studies using six trials allow clusters of up to 17 items; 12 trials allow clusters of up to 9 items. Both variables (number of trials, maximum number of clusters) are input parameters. Program dimensions may be adjusted simply by altering the DIMENSION and COMMON statements at the beginning of the program.

Availability. Program listing is available upon request.

\section{REFERENCES}

BuschKe, H. Learning is organized by chunking. Journal of Verbal learning and Verbal Behavior, 1976, in press.

Pellegrino, J. W. A general measure of organization in free recall for variable unit size and internal sequential consistency. Behavior Research Methods \& Instrumentation, 1971. 3. 241-246.

Pellegrino, J. W. A FORTRAN IV program for analyzing higher order subjective organization units in free recall learning. Behavior Research Methods \& Instrumentation, 1972, 4, 215-217. 